\title{
Low Energy QCD Measurement with NA48/2 at CERN
}

\section{Brigitte Bloch-Devaux*}

Department of Physics, Università degli Studi di Torino,

via P.Giuria 1, 10125 Torino, Italy

E-mail: brigitte.bloch-devauxecern.ch

The NA48/2 collaboration has analyzed 1.13 million charged kaon decays $K^{ \pm} \rightarrow \pi^{+} \pi^{-} e^{ \pm} v$ leading to an improved determination of the branching ratio at percent level precision and detailed form factor studies. The hadronic form factors in the S- and P-wave and their variation with energy are obtained concurrently with the phase difference between the S- and P-wave states of the $\pi \pi$ system. The latter measurement allows a precise determination of $a_{0}^{0}$ and $a_{0}^{2}$, the $I=0$ and $I=2$ S-wave $\pi \pi$ scattering lengths. A combination of this result with another NA48/2 measurement, obtained in the study of $K^{ \pm} \rightarrow \pi^{0} \pi^{0} \pi^{ \pm}$decays, brings a further improved determination of $a_{0}^{0}$ and the first precise experimental measurement of $a_{0}^{2}$. These measurements bring new inputs to low energy QCD studies and are crucial tests of existing predictions from Chiral Perturbation Theory and lattice QCD calculations.

2013 Kaon Physics International Conference,

29 April-1 May 2013

University of Michigan, Ann Arbor, Michigan - USA

\footnotetext{
* Speaker.

† On behalf of the NA48/2 collaboration: Cambridge, CERN, Chicago, Dubna, Edinburgh, Ferrara, Florence, Mainz, Northwestern, Perugia, Pisa, Saclay, Siegen, Turin, Vienna
} 


\section{Introduction}

Kaon decays have been identified as a perfect laboratory to study low energy strong interaction. In the past 35 years the $K_{e 4}$ decay mode $\left(K^{ \pm} \rightarrow \pi^{+} \pi^{-} e^{ \pm} v\right)$ was traditionally the cleanest process to study $\pi \pi$ scattering close to threshold and extract the values of the S-wave scattering lengths $a_{0}^{0}$ and $a_{0}^{2}$ in the $I=0$ and $I=2$ isospin states. Three experiments have collected sizable samples of $K_{e 4}$ decays: the NA48/2 collaboration at the CERN/SPS has published results based on a sample of 1.1 million $K_{e 4}^{ \pm}$decays [1], the E865 collaboration at BNL has analyzed about 400000 such $K_{e 4}^{+}$ decays [2] and the Geneva-Saclay collaboration (S118) at the CERN/PS $30000 K_{e 4}^{+}$decays [3].

A very precise study of the hadronic decay $K_{3 \pi}\left(K^{ \pm} \rightarrow \pi^{0} \pi^{0} \pi^{ \pm}\right)$close to the $2 m_{\pi^{+}}$threshold brings another access to the $\pi \pi$ scattering lengths $\left(a_{0}^{0}-a_{0}^{2}\right)$ and $a_{0}^{2}$ via charge exchange rescattering effects in the final state. A sample of 60 millions such decays has been studied by the NA48/2 collaboration [ $\left[\right.$ ]. The $K_{3 \pi}$ scattering lengths results will be combined with those obtained from the $K_{e 4}$ decay study.

A third complementary approach has been developed by the DIRAC collaboration at CERN [5] by measuring the $\pi \pi$ atoms (pionium) life time and is also reported at this Conference [6]. It brings an independent measurement of $\left|a_{0}^{0}-a_{0}^{2}\right|$.

All three approaches require a close collaboration with theorists to describe precisely the studied reactions.

\section{The NA48/2 Experimental setup}

Two simultaneous intense $K^{+}$and $K^{-}$beams were produced by $400 \mathrm{GeV} / c$ primary CERN/SPS protons, impinging on a beryllium target. The beams were then deflected in a front-end achromat to select momenta in the range $(60 \pm 3) \mathrm{GeV} / \mathrm{c}$ and focused at $\sim 200 \mathrm{~m}$ downstream in front of the first spectrometer chamber. A schematic view of the beam line and detector can be found in Ref. [7]. At the entrance of the decay volume, a $114 \mathrm{~m}$ long vacuum tank, the kaon beams contain $\sim 2.3 \times 10^{6} \mathrm{~K}^{+}$and $\sim 1.3 \times 10^{6} \mathrm{~K}^{-}$per pulse of $4.5 \mathrm{~s}$ duration leading to a flux ratio $\mathrm{K}^{+} / \mathrm{K}^{-}$close to 1.8. The NA48 detector and its performances are described with full details elsewhere [8].

The main components used in the $K_{e 4}$ and $K_{3 \pi}$ analyses are:

- a magnetic spectrometer consisting of a dipole magnet surrounded by two sets of drift chambers achieving a momentum resolution $\sigma(p) / p=(1.02 \oplus 0.044 p) \%(p$ in $\mathrm{GeV} / c)$;

- a 27 radiation length liquid krypton calorimeter $(\mathrm{LKr})$ measuring electromagnetic energy deposits and identifying electrons through their $E / p$ ratio (the transverse segmentation into 13248 projective cells gives an energy resolution $\sigma(E) / E=(3.2 / \sqrt{E} \oplus 9.0 / E \oplus 0.42) \%(\mathrm{E}$ in $\mathrm{GeV})$ and a space resolution for isolated showers $\left.\sigma_{x}=\sigma_{y}=(0.42 / \sqrt{E} \oplus 0.06) \mathrm{cm}\right)$;

- a two plane segmented scintillator hodoscope triggering the detector readout on charged track topologies with a time resolution $\sim 150 \mathrm{ps}$;

- a two-level trigger logic selecting concurrently events with at least two tracks coming from a common vertex and events with a single track and energy deposits in the $\mathrm{LKr}$ calorimeter consistent with at least 2 photons. 


\section{The $K_{3 \pi}$ analysis}

The analysis of a sample of $60.3 \cdot 10^{6}$ decays has been reported in [4]. The $K^{ \pm} \rightarrow \pi^{0} \pi^{0} \pi^{ \pm}$ final state is fully reconstructed from one charged particle measured in the spectrometer and four photons, identified from their energy deposits in the LKr calorimeter and forming two $\pi^{0}$ s pointing to the same decay vertex. The reconstructed invariant mass of the three pions matches the kaon nominal mass with a resolution of $1.3 \mathrm{MeV} / \mathrm{c}^{2}$ (Fig. 1 l left). The invariant mass squared of the two $\pi^{0} \mathrm{~s}\left(M_{00}^{2}\right)$ exhibits a cusp-like behavior around the $2 m_{\pi^{+}}$threshold (Fig.1 1 right).
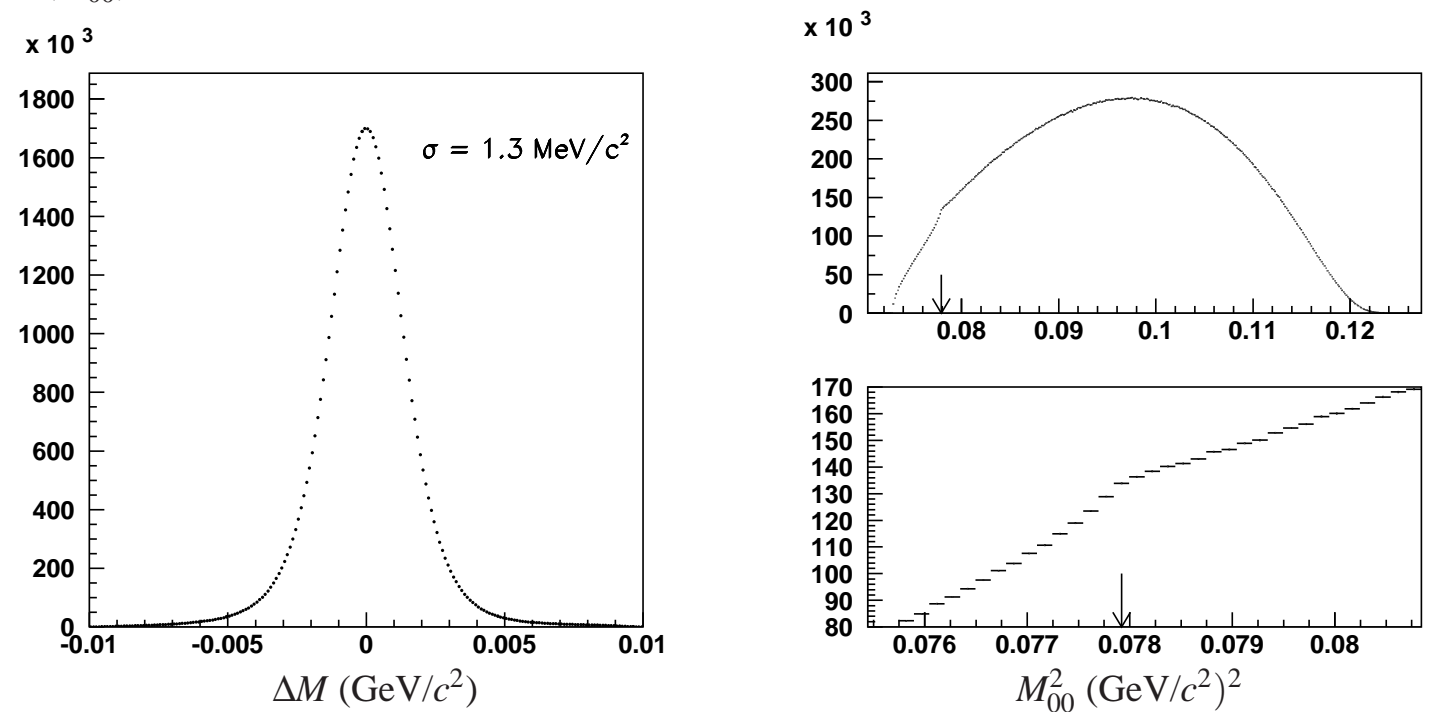

Figure 1: Left: Difference between the reconstructed three pion invariant mass and the nominal kaon mass. Right: The invariant mass squared of the two $\pi^{0} \mathrm{~s}$ is shown on the upper plot. The lower plot is an enlargement of the narrow region around $M_{00}^{2}=4 m_{\pi^{+}}^{2}$ (arrow).

Two theoretical approaches have been considered to determine the scattering lengths from the shape of the $M_{00}^{2}$ distribution:

- a 2-loop diagrams formulation [-1] without radiative corrections;

- an effective Lagrangian approach [10] which includes electromagnetic effects but requires also the simultaneous description of the charged $\left(K^{ \pm} \rightarrow \pi^{+} \pi^{-} \pi^{ \pm}\right)$and neutral $\left(K^{ \pm} \rightarrow \pi^{0} \pi^{0} \pi^{ \pm}\right)$modes form factors.

The complexity of the above descriptions makes the close collaboration with theorists absolutely mandatory. The results are obtained from a simultaneous fit of the form factors, the scattering lengths and a contribution from pionium at its formation threshold. The obtained scattering lengths in $m_{\pi^{+}}$unit are:

$$
a_{0}^{0}-a_{0}^{2}=0.2571 \pm 0.0048_{\text {stat }} \pm 0.0029_{\text {syst }}, a_{0}^{2}=-0.0241 \pm 0.0129_{\text {stat }} \pm 0.0096_{\text {syst }},
$$

with -0.88 correlation between the two fit-parameters.

\section{The $K_{e 4}$ analysis}

The analysis of a total data sample of $1.1 \cdot 10^{6} K^{ \pm} \rightarrow \pi^{+} \pi^{-} e^{ \pm} V$ candidates with $\sim 1 \%$ background has been reported in [1, 11]. The events are reconstructed from three charged tracks coming 
from the same decay vertex with missing mass and missing transverse momentum with respect to a $K_{3 \pi}$ decay hypothesis. The selection requires one charged track to be identified as an electron if the $E / p$ ratio of its energy $E$, measured in the $\mathrm{LKr}$ calorimeter, and its momentum $p$, measured in the spectrometer, is close to unity.

The $K_{e 4}$ decay is fully described by the five kinematic Cabibbo-Maksymowicz variables [12] (two invariant masses: $S_{\pi}=M_{\pi \pi}^{2}$ and $S_{e}=M_{e v}^{2}$, and three angles: $\theta_{\pi}, \theta_{e}$ and $\phi$, characterizing the kinematic in (and between) the dipion and dilepton planes). Form factors are then developed in a partial wave expansion [13]. When limiting the expansion to S- and P-waves and considering a unique phase $\delta_{p}$ for all P-wave form factors, two complex axial $(F, G)$ and one complex vector $(H)$ form factors contribute to the transition amplitude:

$$
F=F_{s} e^{i \delta_{s}}+F_{p} e^{i \delta_{p}} \cos \theta_{\pi}, G=G_{p} e^{i \delta_{p}}, H=H_{p} e^{i \delta_{p}} .
$$

Under the assumption of T-invariance, the phase $\delta_{p}$ is further identified with the phase $\delta_{1}^{1}$ of the $\pi \pi$ scattering amplitude while $\delta_{s}$ can be identified to $\delta_{0}^{0}$ if the additional $\Delta I=\frac{1}{2}$ rule in semileptonic decays holds [13]. This property has been used until recently in all $K_{e 4}$ analyses [3, 2, 14].

The sample is distributed over a grid of 15000 equal population boxes in the five-dimensional space. A simultaneous fit is performed in each of the ten $S_{\pi}$ bins to obtain four real form factors $\left(F_{s}, F_{p}, G_{p}\right.$ and $\left.H_{p}\right)$ and a single phase $\left(\delta=\delta_{s}-\delta_{p}\right)$, without prior assumption on the shape of their variation with $S_{\pi}, S_{e}$. The scattering lengths are then obtained by inverting the numerical solutions of the Roy equations [15] which relate the phases of the $\pi \pi$ scattering $\delta_{0}^{0}, \delta_{1}^{1}$ close to threshold to the $\mathrm{S}$-wave scattering lengths in the $I=0$ and $I=2$ isospin states, $a_{0}^{0}, a_{0}^{2}$.

New theoretical works [16] have shown that isospin symmetry breaking may also alter the phases measured in $K_{e 4}$ decay when all mass effects $\left(m_{\pi^{+}} \neq m_{\pi^{0}}, m_{u} \neq m_{d}\right)$, neglected so far in previous analyses, are considered. Even if the difference between the isospin-symmetric phase and the modified phase is modest in terms of absolute magnitude (10 to $15 \mathrm{mrad}$ ) over the whole accessible mass range (between threshold and $M_{\pi \pi}=0.39 \mathrm{GeV} / c^{2}$ ), the coherent shift toward higher values of the precisely measured phases has non negligible implications when extracting scattering lengths from such measurements as shown in Figure 2 The results obtained using [16] are:

$$
\begin{aligned}
& a_{0}^{0}=0.2220 \pm 0.0128_{\text {stat }} \pm 0.0050_{\text {syst }} \pm 0.0037_{\text {th }}, \\
& a_{0}^{2}=-0.0432 \pm 0.0086_{\text {stat }} \pm 0.0034_{\text {syst }} \pm 0.0028_{\text {th }},
\end{aligned}
$$

with 0.97 correlation between the two fit parameters. The quoted theory uncertainty comes from the precision on the external inputs (Roy equations) and the neglected higher order terms (isospin corrections). Using another calculation of the corrections [17], based on similar effects on the phases of the pion scalar and vector form factors, does not change significantly the results. A new dispersive approach to isospin breaking effects in $K_{e 4}$ form factors has been reported at this conference [18] and subsequently published [19] resulting in perfectly consistent values of $a_{0}^{0}, a_{0}^{2}$ scattering lengths when applied to our phase measurements.

\section{Combining scattering lengths results}

The $K_{3 \pi}$ and $K_{e 4}$ results are obviously statistically independent. They have systematic uncertainties of different origins and show different correlations between the fitted scattering lengths. 

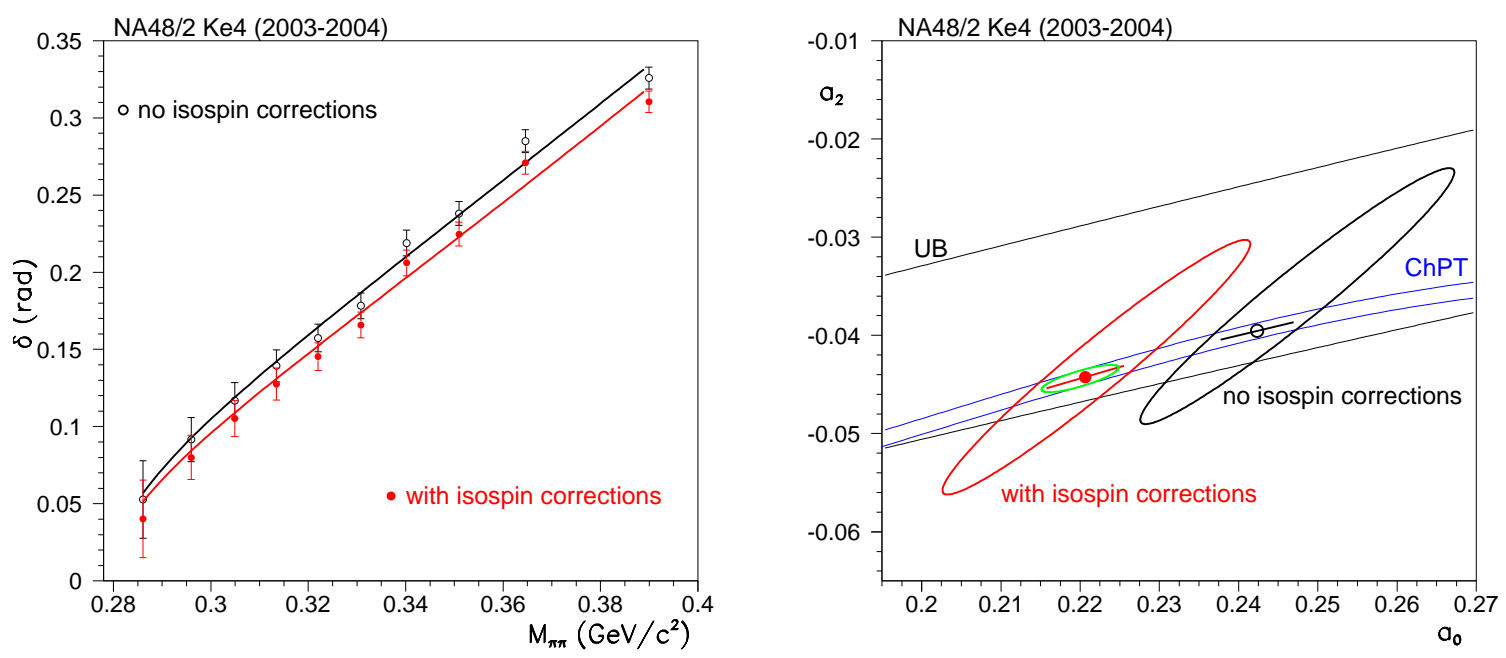

Figure 2: Left: Phase difference $\delta$ between the S- and P-wave form factors. Measurements are shown without (open circles) and with (full circles) isospin mass effects corrections. The lines correspond to the two-parameter fit using Roy equations solutions from [15]. Errors are statistical only. Right: Fits to the $K_{e 4}$ data in the $\left(a_{0}^{0}, a_{0}^{2}\right)$ plane without (black contour) and with (red contour) isospin mass effects corrections. Errors are statistical only, contours are $68 \% \mathrm{CL}$. The symbols with error bars are the results of a oneparameter fit, imposing the ChPT constraint [20]. The smaller green ellipse corresponds to the best prediction from ChPT [21] $\left(a_{0}^{0}=0.220 \pm 0.005, a_{0}^{2}=-0.0444 \pm 0.0010\right)$.

Neglecting potential (but small) common systematic contribution to the experimental errors, it is possible to combine the two measurements (Eq. 3.1 and 4.1) in a more precise result:

$$
\begin{array}{rr}
a_{0}^{0}=0.2210 \pm 0.0047_{\text {stat }} \pm 0.0040_{\text {syst }}, \\
a_{0}^{2}=-0.0429 \pm 0.0044_{\text {stat }} \pm 0.0028_{\text {syst }}, \\
a_{0}^{0}-a_{0}^{2}=0.2639 \pm 0.0020_{\text {stat }} \pm 0.0015_{\text {syst }} .
\end{array}
$$

The two input sets of values and their combination are displayed in Figure 3 together with the most recent result from DIRAC [6], the precise calculations of lattice QCD collaborations [22, 23] and the most elaborate prediction from ChPT [21].

\section{Branching ratio and form factors in the $K_{e 4}$ decay mode}

The energy dependence of the form factors have been studied in the plane $\left(S_{\pi}, S_{e}\right)$ and obtained at few percent precision [1]. All values are measured relative to a common constant $f_{s}$ determined from the branching ratio measurement [11]. The main features of the results are the evidence for a constant 5\% contribution of $F_{p}$, the linear variation of $G_{p}$ with $S_{\pi}$ and the quadratic variation with $S_{\pi}$ and linear variation with $S_{e}$ of $F_{s}$ in the $\left(S_{\pi}, S_{e}\right)$ plane.

The branching ratio is measured relative to the abundant mode $K_{3 \pi}\left(K^{ \pm} \rightarrow \pi^{+} \pi^{-} \pi^{ \pm}\right)$which has the same three-track topology as the $K_{e 4}$ signal mode and is recorded concurrently through the same trigger logic, minimizing systematic effects which partially cancel between signal and normalization:

$$
\operatorname{BR}\left(K_{e 4}\right)=\frac{N_{s}-N_{b}}{N_{n}} \cdot \frac{A_{n} \varepsilon_{n}}{A_{s} \varepsilon_{s}} \cdot \operatorname{BR}(n)
$$



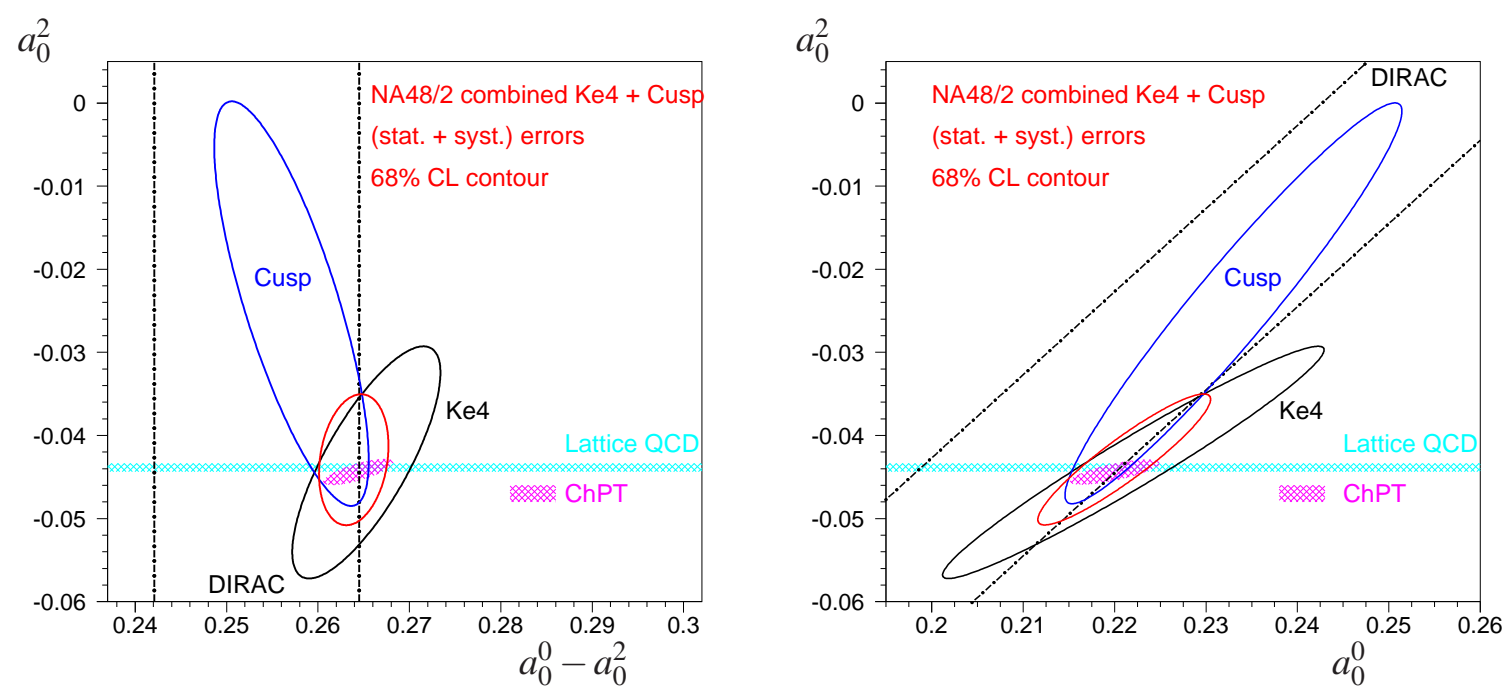

Figure 3: NA48/2 $K_{e 4}$ (black) and $K_{3 \pi}$ (blue) results from the two-parameter fits in the $\left(a_{0}^{0}-a_{0}^{2}, a_{0}^{2}\right)$ (left) and $\left(a_{0}^{0}, a_{0}^{2}\right)$ (right) planes. In each plane the smallest (red) contour corresponds to the combination of the NA48/2 results. The correlation coefficient is then 0.21 in the left plane and 0.92 in the right plane. The dashed lines display the DIRAC result band. The colored horizontal band corresponds to the lattice QCD calculations of $a_{0}^{2}$ and the hatched ellipse to the most precise prediction [21] from ChPT for $a_{0}^{0}, a_{0}^{2}$.

where $N_{s}, N_{b}, N_{n}$ are the numbers of signal, background and normalization candidates, $A_{s}, \varepsilon_{s}\left(A_{n}, \varepsilon_{n}\right)$ are the geometrical acceptance and trigger efficiency for the signal (normalization) sample, respectively. The normalization branching ratio $\operatorname{BR}(n)=(5.59 \pm 0.04) \%$ is the world average from [24].

The final result is a weighted average of 16 values obtained in eight independent data subsamples and for both kaon charges. It corresponds to $1.1 \cdot 10^{6}$ signal candidates with less than $1 \%$ relative background and $1.9 \cdot 10^{9}$ normalization events. Geometrical acceptances are $O(20 \%)$ and trigger efficiencies about $98 \%$. The resulting value, including all errors, is:

$$
\operatorname{BR}\left(K_{e 4}\right)=\left(4.257 \pm 0.004_{\text {stat }} \pm 0.016_{\text {syst }} \pm 0.031_{\text {ext }}\right) \times 10^{-5}
$$

where the error is dominated by the external uncertainty from the normalization mode. The $\operatorname{BR}\left(K_{e 4}\right)$ values obtained for the statistically independent subsamples are shown in Fig. $\theta$.

The $K_{e 4}$ branching ratio, inclusive of radiative decays, can also be expressed as:

$$
\operatorname{BR}\left(K_{e 4}\right)=\tau_{K^{ \pm}} \cdot\left(\left|V_{u s}\right| \cdot f_{s}\right)^{2} \cdot I_{5},
$$

where $\tau_{K^{ \pm}}$is the $K^{ \pm}$mean lifetime (in seconds) and $I_{5}$ is the integrated differential rate over the 5dimensional space after substituting the form factors by their measured values, including radiative effects and leaving out the $\left|V_{u s}\right|$ and $f_{s}$ constants. The value of $f_{s}$ is then obtained from the measured value of $\mathrm{BR}\left(K_{e 4}\right)$ and the integration result. Using (6.1) and the world average kaon lifetime value $(1.2380 \pm 0.0021) \times 10^{-8} \mathrm{~s}$, the measurement of the form factors is now complemented by the overall $f_{s}$ normalization:

$$
\begin{aligned}
\left|V_{u s}\right| \cdot f_{s} & =1.285 \pm 0.001_{\text {stat }} \pm 0.004_{\text {syst }} \pm 0.005_{\text {ext }} \\
\text { corresponding to } f_{s} & =5.705 \pm 0.003_{\text {stat }} \pm 0.017_{\text {syst }} \pm 0.031_{\text {ext }}
\end{aligned}
$$

when using $\left|V_{u s}\right|=0.2252 \pm 0.0009$ [24]. Table 1 displays all form factor values obtained at improved precision. 


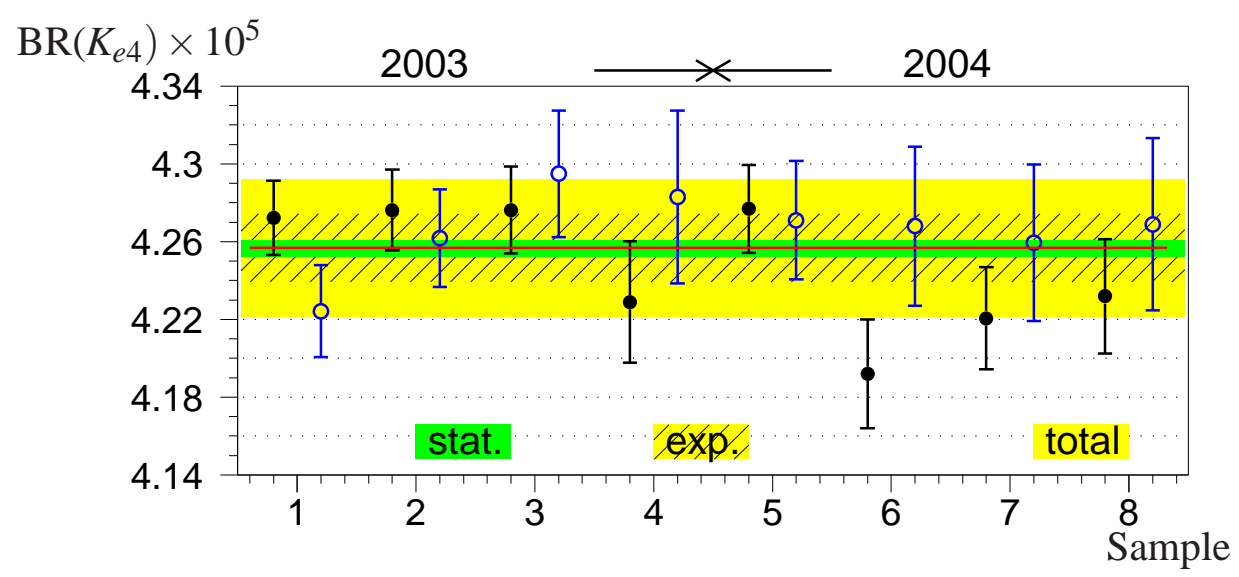

Figure 4: $K_{e 4}$ branching ratio for eight statistically independent samples and both kaon charges. The hatched band shows the experimental error $\left(\sigma_{\text {exp }}=\sigma_{\text {stat }} \oplus \sigma_{\text {syst }}\right)$. The total error (shaded band) includes the external error. Full symbols correspond to $K^{+}$results and empty symbols to $K^{-}$results.

Table 1: Absolute values of the measured form factors. Their variation in the $\left(S_{\pi}, S_{e}\right)$ plane is parameterized as a series expansion of the dimensionless variables $q^{2}\left(q^{2}=S_{\pi} / 4 m_{\pi}^{2}-1\right)$ and $S_{e} / 4 m_{\pi}^{2}$. There are large anticorrelations between $f_{s}^{\prime}, f_{s}^{\prime \prime}(-0.954)$ and $g_{p}, g_{p}^{\prime}(-0.914)$. The normalization error is fully correlated over all form factors.

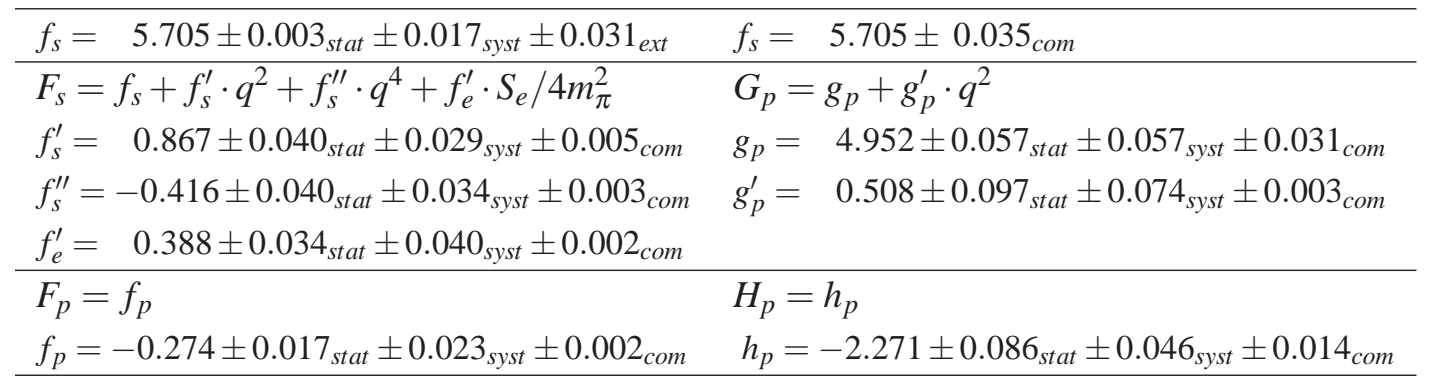

\section{Summary}

The NA48/2 experiment has produced a wide collection of results providing precision tests of low energy QCD. High precision measurements of $K_{e 4}$ and $K_{3 \pi}$ decay modes lead to converging results for the $S$-wave $\pi \pi$ scattering lengths $a_{0}^{0}$ and $a_{0}^{2}$, in very good agreement with ChPT predictions and lattice QCD calculations. This is the first experimental measurement of the scattering length $a_{0}^{2}$. The most precise $K_{e 4}$ branching ratio ( $0.8 \%$ relative) has been obtained allowing absolute form factor determination with evidence for a small $F_{p}$ contribution and a $\left(S_{\pi}, S_{e}\right)$ dependence of $F_{s}$.

\section{References}

[1] NA48/2: J. Batley et al., Eur. Phys. J. C70 (2010) 635.

[2] E865: S. Pislak et al., Phys. Rev. D67 (2003) 072004.

[3] S118: L. Rosselet et al., Phys. Rev. D15 (1977) 574.

[4] NA48/2: J. Batley et al., Eur. Phys. J. C64 (2009) 589.

[5] DIRAC: Phys. Lett. B704 (2011) 24. 
[6] L. Nemenov, this conference, PoS(KAON13) 024.

[7] NA48/2: J. Batley et al., Eur. Phys. J. C52 (2007) 875.

[8] NA48: V. Fanti et al., Nucl. Instrum. Methods A574 (2007) 433.

[9] N. Cabibbo, G. Isidori, J. High Energy Phys. 03 (2005) 021.

[10] M. Bissegger, A. Fuhrer, J. Gasser, B. Kubis, A. Rusetsky, Nucl. Phys. B806 (2009) 178.

[11] NA48/2: J. Batley et al., Phys. Lett. B715 (2012) 105.

[12] N. Cabibbo and A. Maksymowicz, Phys. Rev. B438 (1965) 137.

[13] A. Pais and S. Treiman, Phys. Rev. 168 (1968) 1858.

[14] NA48/2: J. Batley et al., Eur. Phys. J. C54 (2008) 411.

[15] B. Ananthanarayan, G. Colangelo, J. Gasser, H. Leutwyler, Phys. Rep. B353 (2001) 207.

[16] G. Colangelo, J. Gasser and A. Rusetsky, Eur. Phys. J. C59 (2009) 777.

[17] S. Descotes-Genon, M. Knecht, Eur. Phys. J. C72 (2012) 1962.

[18] S. Descotes-Genon, this conference, PoS(KAON13) 022.

[19] V. Bernard, S. Descotes-Genon, M. Knecht, arXIv:1305.3843[hep-ph], accepted for publication in Eur. Phys. J. C (2013) .

[20] G. Colangelo, J. Gasser, H. Leutwyler, Phys. Rev. Lett. 86 (2001) 5008.

[21] G. Colangelo, J. Gasser, H. Leutwyler, Phys. Lett. B488 (2000) 261.

[22] NPLQCD: S. Beane et al., Phys. Rev. D77 (2008) 014505.

[23] ETM: X. Feng, K. Jansen, D. Renner, Phys. Lett. B684 (2010) 268.

[24] PDG: J. Beringer et al., Phys. Rev. D86 (2012) 010001. 